\title{
Dispositifs interocclusaux et prise en charge des DTM
}

\section{RÉSUMÉ}

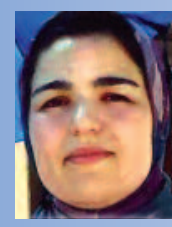

\section{Anissa ABDELKOUI}

Chirurgien-dentiste,

Résidente en prothèse adjointe

Unité occlusodontie,

Faculté de médecine dentaire, Rabat,

Avenue Allal El Fassi, rue Mohammed Jazouli,

Cité Madinat Al Irfane,

BP 6212, Rabat-Instituts, Maroc

\section{Leila FAJRI}

Professeur assistant en prothèse adjointe.

\section{Ahmed ABDEDINE}

Professeur de l'enseignement supérieur en prothèse adjointe et en occlusodontie, Chef de service de prothèse adjointe, Faculté de médecine dentaire, Rabat.

Les dispositifs interocclusaux, initialement utilisés pour protéger les dents et les articulations temporomandibulaires en cas de bruxisme, sont actuellement indiqués dans de nombreuses situations cliniques.

Ces appareils, souvent indiqués pour les dysfonctionnements temporomandibulaires (DTM), entraînent une amélioration incontestable de l'état des patients souffrant de ces pathologies.

À travers cet article, nous essaierons de passer en revue les différents types de dispositifs interocclusaux, de définir leurs rôles et leurs objectifs, puis de déterminer pour chacun la procédure de réalisation afin de pouvoir faire un choix judicieux, le mieux adapté à la prise en charge des DTM.

- dispositifs interocclusaux

- désordres temporomandibulaires

- système manducateur

- articulation temporomandibulaire
AOS 2011;255:243-253

DOI: $10.1051 / a 0 s / 2011307$

(C) AEOS / EDP Sciences 


\section{Introduction} $>\begin{aligned} & \text { Les dispositifs interocclusaux sont des } \\ & \text { appareillages orthopédiques intra-oraux }\end{aligned}$ qui, placés à l'arcade maxillaire ou mandibulaire, modifient de façon transitoire ou définitive l'intercuspidation maximale.

Ils comprennent les butées occlusales antérieures, les butées postérieures, les plans de morsures, les différents types de gouttières

\section{Principes théoriques}

Plusieurs explications au bénéfice des dispositifs interocclusaux ont été avancées.

\section{Effet placebo}

\section{et prise de conscience}

des contacts occlusaux

Les dispositifs interocclusaux présentent, comme de nombreuses thérapeutiques, un effet placebo. En fait, la simple mise en place de l'un de ces appareillages dans la cavité buccale rassure le patient, en lui montrant que le praticien s'occupe de son problème.

Le dispositif interocclusal matérialise donc le traitement cognitif qui a été induit par les explications données par le praticien.

Il rappelle constamment au patient qu'il doit éviter de serrer les dents, qu'il doit supprimer certaines habitudes parafonctionnelles et, par conséquent, diminuer son activité musculaire [2].

\section{Rétablissement de la DVO}

Il est actuellement bien reconnu que la diminution de la DVO s'accompagne d'une hyperactivité musculaire au niveau des muscles élévateurs, occlusales et les appareillages d'avancée mandibulaire.

Tous ces appareils visent à rétablir un équilibre physiologique ou thérapeutique entre les différents composants du système stomatognathique. Ils peuvent être utilisés à titre de test diagnostique et/ou à titre thérapeutique dans le cadre de la prise en charge des DTM [1].

alors que le rétablissement de celle-ci entraîne une fonction musculaire équilibrée $[3,4]$.

De plus, le rétablissement de la DVO s'accompagne d'un repositionnement des éléments disco- condyliens ainsi que d'une modification de la posture céphalique.

\section{Stabilisation posturale de la mandibule et équilibre occlusal}

La gouttière occlusale permet de compenser des anomalies occlusales telles qu'une perte de la DVO, une perte de calage postérieur, une déviation transversale. En fait, le rétablissement d'un schéma occlusal idéal, sans prématurité ni interférence grâce à une orthèse occlusale, éliminerait les boucles de rétroaction négatives bâties autour de l'ancienne occlusion d'intercuspidie maximale et par conséquent l'activité musculaire serait plus économique $[2,5]$.

\section{Position de réalignement}

Trois approches sont proposées pour déterminer la position de réalignement. 
Selon Dawson et les gnathologistes, la position de relation centrée est considérée comme la position de référence. Elle est obtenue par la manipulation de la mandibule en cherchant une ouverture buccale reproductible en rotation pure et un réalignement des pièces articulaires [6].

Jankelson propose la position d'équilibre musculaire qui dépend uniquement de l'action des muscles abaisseurs, élévateurs et posturaux.

Jeanmonod favorise la relation intermaxillaire myodéterminée grâce à un plan de morsure rétro-incisif qui, en supprimant les engrammes des malocclusions, réduirait l'activité musculaire et donc faciliterait l'obtention d'une relation centrée myostabilisée $[2,7]$.
Lieb, quant à lui, a recours à une position qui prend en compte des repères anatomiques variés au niveau des dents, des procès alvéolaires et des axes de symétrie, pour définir le réalignement qui sert de référence à la confection du dispositif interocclusal $[2,4]$.

\section{Repositionnement condylien}

Dans certaines situations où le disque articulaire est déplacé ou altéré, le dispositif interocclusal vise la décompression de l'articulation temporomandibulaire et/ou la stabilisation de la mandibule dans une position thérapeutique qui assure la coaptation condylodiscale.

\section{Différents types de dispositifs interocclusaux}

Selon les objectifs et les modes d'action

Selon les objectifs et les modes d'action, on distingue les types suivants :

\section{> Les dispositifs visant à traiter les pathologies musculaires}

On en différencie deux types:

- les appareillages qui couvrent l'arcade en totalité, dont les surfaces sont lisses, et qui permettent, en supprimant les contacts dento-dentaires, d'éliminer les boucles de rétroactions négatives favorisant ainsi une activité musculaire synchrone et optimale [8] ;

- les dispositifs qui couvrent partiellement l'arcade dentaire (le jig, plan de morsure), qui ont pour objectif de supprimer toutes les afférences proprioceptives au niveau des secteurs postérieurs, en créant une désocclusion minimum à ce niveau ;

\section{> Les dispositifs visant à traiter les pathologies articulaires}

Ces dispositifs ont pour simple vocation le repositionnement mandibulaire afin de rétablir une coaptation discale et/ou d'effectuer une décompression de l'ATM. Leur surface est indentée de façon à guider la mandibule dans une nouvelle intercuspidation prenant en compte la position articulaire thérapeutique.

\section{Selon l'efficacité clinique}

Selon l'efficacité clinique, on distingue par ordre décroissant les types suivants : 


\section{> Gouttière de reconditionnement}

Appelée également gouttière de relaxation ou de libération neuromusculaire, elle est réalisée en résine acrylique dure, lisse, de préférence transparente, recouvrant la totalité de l'arcade maxillaire ou mandibulaire.

Cette gouttière, en étant plane, supprime les boucles de rétroaction négatives ce qui rendrait l'activité musculaire plus équilibrée.

Elle est utilisée pour protéger les dents en cas de bruxisme, en cas de problèmes musculaires ou en l'attente d'un diagnostic plus précis $[3,5]$.

\section{- Réalisation}

Cette gouttière est confectionnée au laboratoire à partir de modèles en plâtre des arcades dentaires, montés sur un articulateur semi-adaptable par l'intermédiaire de l'arc facial, permettant de conserver comme référence le plan axio-orbitaire tout au long du traitement (fig. 1).

Lors de la mise en place de la gouttière, celle-ci se doit d'être stable, rétentive par elle-même et ne présenter ni prématurité ni interférence, mais des contacts occlusaux simultanés avec l'arcade antagoniste, répartis sur l'ensemble de la gouttière (fig. 2).

Afin d'organiser les mouvements mandibulaires excursifs, le respect du guide antérieur et canin est impératif.

La gouttière doit être compatible avec une DVO confortable pour le patient.

Cette orthèse doit être portée la nuit ou pendant certaines activités (professionnelles, sportives, etc.) et rectifiée tous les 15 jours pour suivre l'évolution de la position mandibulaire au fur et à mesure que la pathologie musculaire disparaît.

Autre technique : la gouttière peut être également réalisée à partir d'une plaque thermoformée ramollie à chaud et plaquée sur le modèle de travail.

Après refroidissement, la face occlusale est façonnée par adjonction de résine auto-polymérisable.
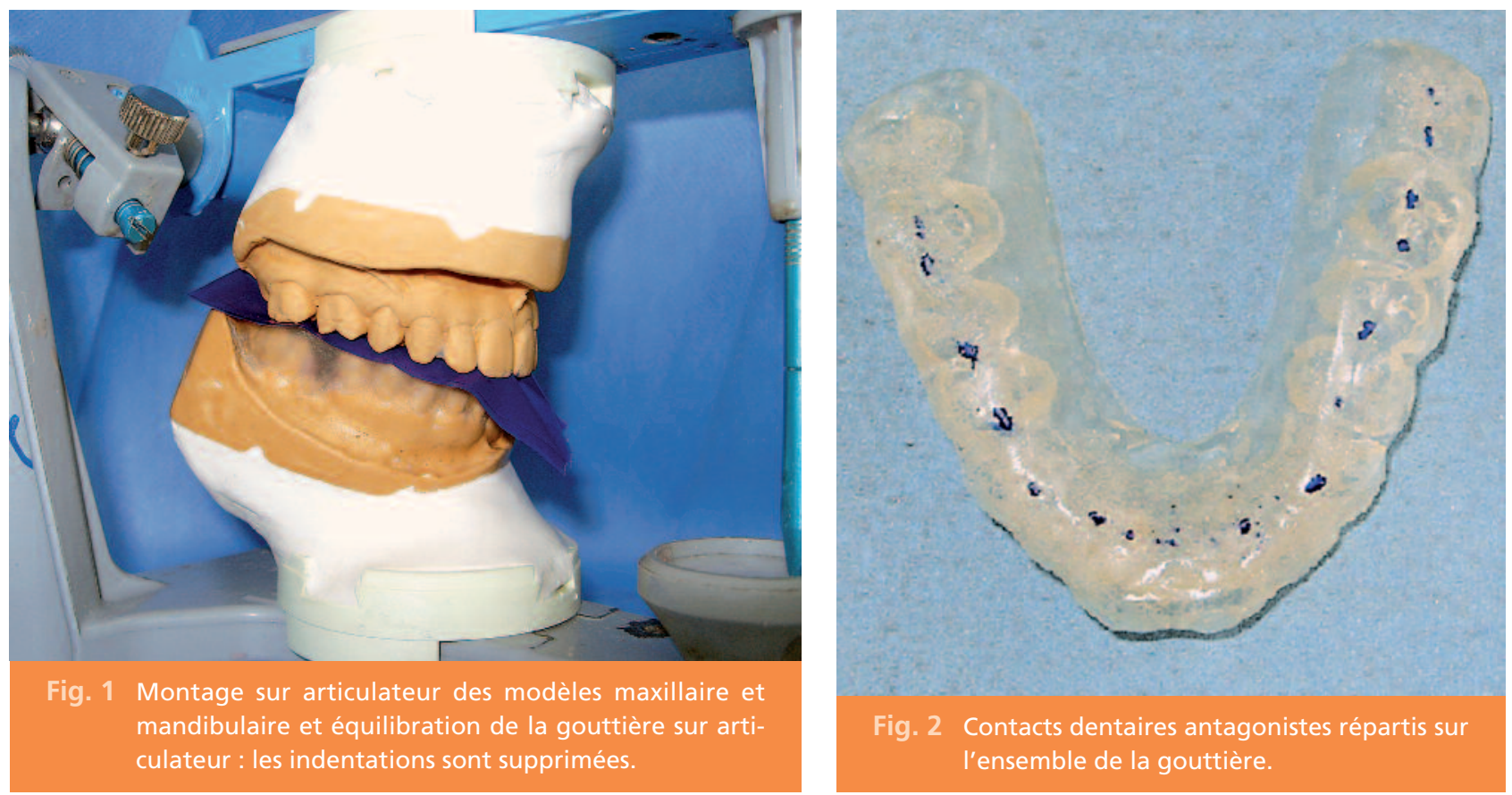


\section{$>$ Gouttière de protection ou antistress}

Chez les patients bruxomanes, les parafonctions provoquent la destruction des structures dentaires de calage, de centrage et de guidage et créent des facettes d'abrasion qui deviennent des facteurs d'entretien du bruxisme [9].

Pour l'odontologiste, le standard du traitement du bruxisme consiste en le port d'une orthèse occlusale qui permet à la fois de protéger les dents et les ATM, en redistribuant les forces occlusales et éventuellement de limiter les effets des contractions musculaires.

Cette orthèse occlusale, dite de protection ou " antistress ", répond aux mêmes caractéristiques de réalisation que la gouttière de relaxation musculaire (fig. 3) [10].

Elle doit être portée durant toute la journée en cas de bruxisme diurne ou pendant la nuit lorsque le bruxisme est nocturne.

Dans le cas où la dimension verticale d'occlusion (DVO) est diminuée, on peut réaliser une gouttière dite de " verticalisation " qui ressemble aux précédentes, avec une épaisseur rétablissant la DVO. Cette gouttière permet de tester et de valider la nouvelle position mandibulaire avant un éventuel traitement stabilisa- teur, tout en assurant un rôle de protection des structures de l'appareil manducateur.

\section{> Gouttière de repositionnement}

Les troubles articulaires résultent en grande partie d'un déplacement vers l'avant du disque articulaire. Ce déplacement peut être réductible ou irréductible [11, 12].

La gouttière de repositionnement trouve ici son indication et a pour objectif, dans la mesure du possible, de repositionner la mandibule dans une position telle que le disque articulaire soit correctement coapté.

Quand le déplacement discal irréductible est ancien, ce type d'appareillage ne peut être indiqué $[13,14]$.

\section{- Réalisation}

La réalisation de cette gouttière nécessite le montage sur articulateur en relation centrée du jour et la programmation par axiographie.

En fait, il est important de connaître avec exactitude l'endroit précis du repositionnement, l'amplitude de propulsion et l'angulation de celle-ci.

Le tracé axiographique permet la visualisation du déplacement condylien et le réglage de sa

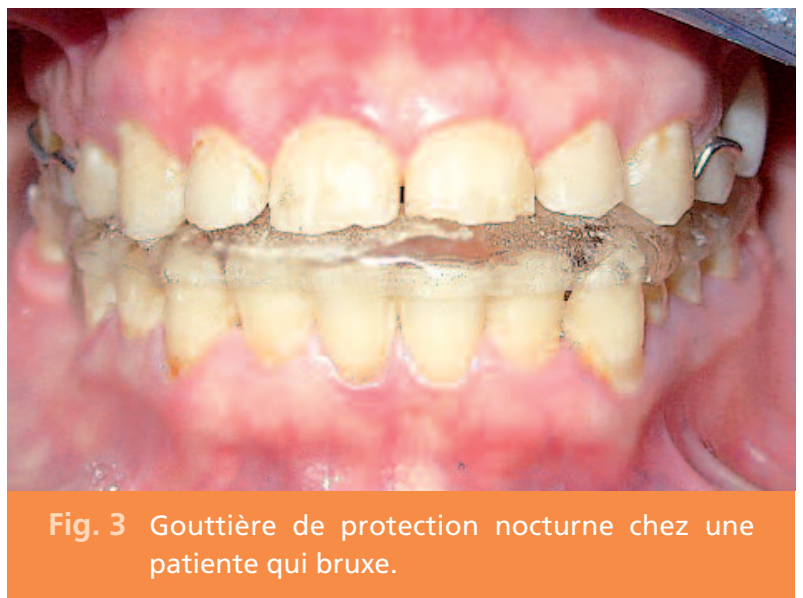


pente. La position de réduction est ainsi programmée sur articulateur, à l'aide de cales de propulsion.

Généralement, la propulsion se fait d'une façon identique des deux côtés. Cependant, on peut envisager de propulser différemment à droite et à gauche.

Cette position thérapeutique peut être également enregistrée directement en bouche à l'aide d'un mordu en cire juste avant le claquement de fermeture.

La tige interincisive est gérée de façon à limiter l'épaisseur de la gouttière et de la maintenir si possible près de l'espace libre d'inocclusion.

La gouttière est indentée (fig. 4 a et b), avec un guidage canin strict (un mur de guidage), évitant ainsi les interférences lors des mouvements excursifs.

Le port doit être permanent et la nouvelle position doit être adoptée spontanément par le patient.

Après trois mois, une tentative de recul mandibulaire par meulage de la rampe et des indentations doit être effectuée.

L'objectif souhaité de cette phase de traitement est de ramener progressivement le complexe condyle-disque dans une position anatomique plus proche de la fosse articulaire.

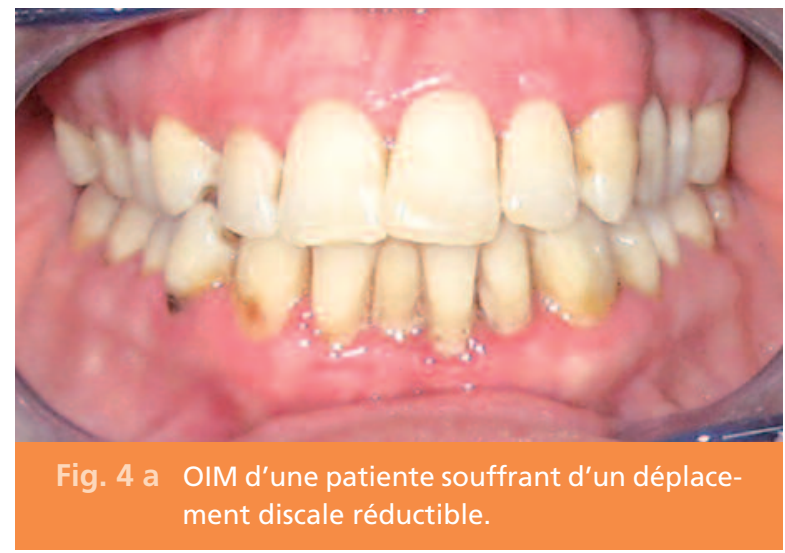

Notons que la réapparition de bruits articulaires doit rendre le praticien très prudent par rapport à la réussite du traitement. En effet, si le patient éprouve des difficultés à conserver la position choisie, il est quelquefois sage, à ce stade, de renoncer au repositionnement articulaire. Dans ce cas, la gouttière de repositionnement articulaire est transformée en gouttière de décompression [15].

\section{> Gouttière de décompression}

La compression articulaire est une conséquence très fréquente des désordres temporomandibulaires. Elle s'accompagne de douleurs souvent invalidantes au niveau de l'ATM, de limitation de la course condylienne et des mouvements mandibulaires, et parfois même de troubles auriculaires...

Lorsque la compression devient chronique, cela peut entraîner une perforation des tissus rétrodiscaux et par conséquent le contact des surfaces osseuses entre elles, pouvant évoluer vers des phénomènes dégénératifs de type arthrosique [16].

Le port d'une gouttière de décompression trouve ici toute sa justification et permet donc de décomprimer l'articulation temporomandibulaire.

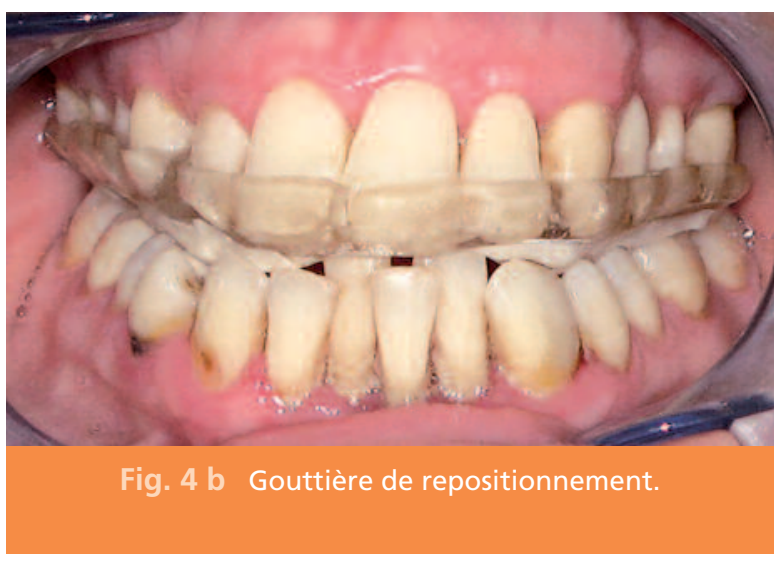


En fait, ce type d'orthèse est essentiellement indiqué en cas de déplacement discal réductible tardif et en cas de déplacement discal non réductible [5].

Il est également utilisé dans les cas d'arthroses dégénératives, d'ostéochondrite et dans les maladies générales ayant une incidence dégénérative sur I'ATM (polyarthrite, lupus) [12].

\section{- Réalisation}

Elle est réalisée dans une position de référence (position thérapeutique) sur articulateur programmé selon les données axiographiques.

Des bandelettes de films radiographiques sont placées dans les boîtiers condyliens pour abaisser la boule condylienne (fig. 5 et 6 ). La tige incisive permet de régler la hauteur de construction de la gouttière.

Lors de la mise en place de ce dispositif, les contacts des dents antagonistes se font simultanément et uniquement au niveau des dernières dents maxillaires (fig. 7). Les dents antérieures sont en légère inocclusion ne dépassant pas $1 \mathrm{~mm}$. Celle-ci induit, lors de la fermeture buccale, une bascule mandibulaire qui décomprime les ATM.

Cette gouttière est également portée 24 heures sur 24, même pendant les repas.

Chaque contrôle est espacé d'au moins une semaine. Ainsi, nous obtenons à terme une descente condylienne verticale pure dans sa fosse glénoïde, libérant ainsi le pincement articulaire existant.

Le maintien de la mandibule dans la position thérapeutique, donnée par la gouttière pendant un temps suffisant (6 à 12 mois), permet d'obtenir une densification fibreuse rétro-discale (néo-disque) [12].

\section{> Butées ou cales postérieures}

Les butées postérieures (fig. 8) présentent un triple objectif : soulager la douleur, recoapter le disque et réadapter les structures articulaires osseuses et ligamentaires en diminuant la pression intracapsulaire.
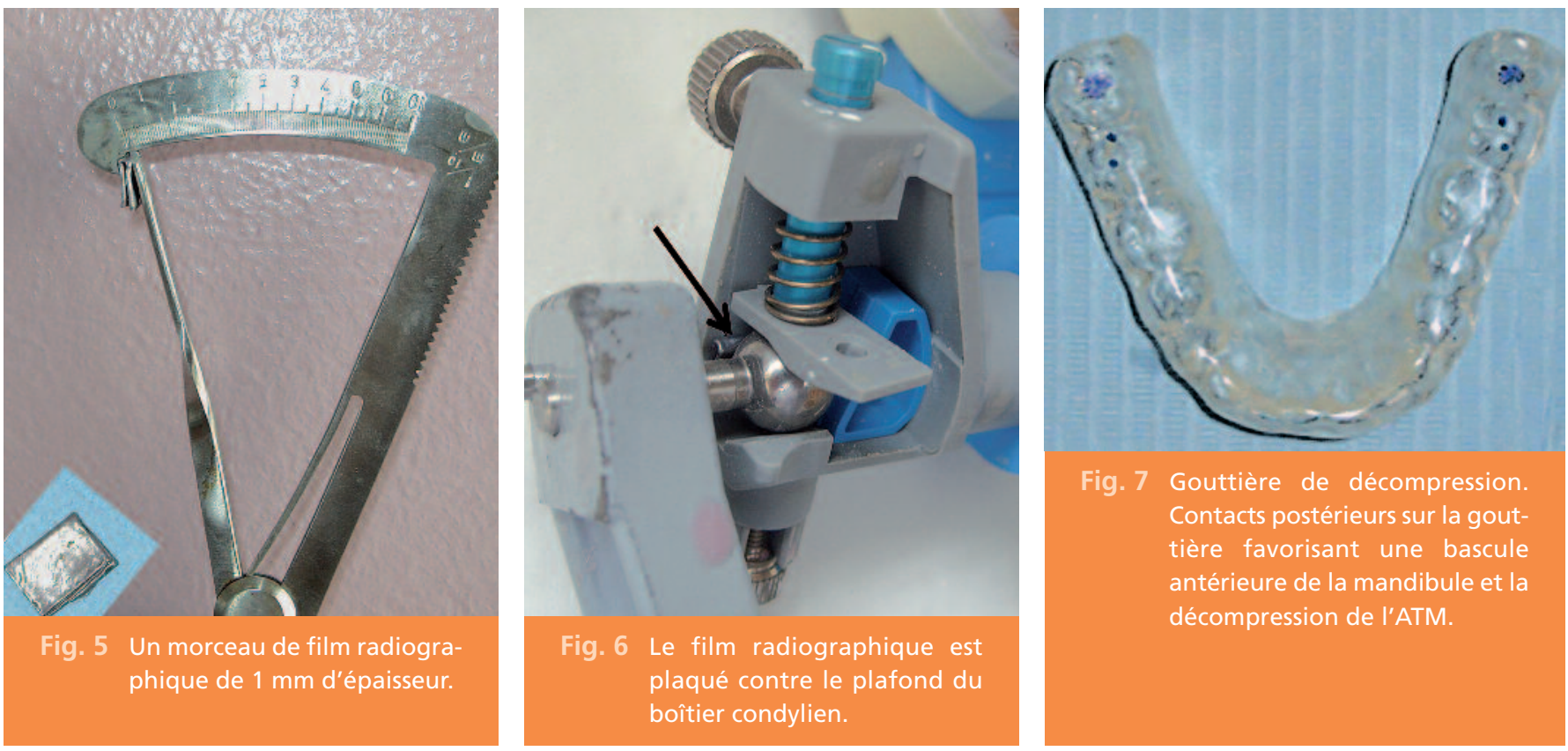


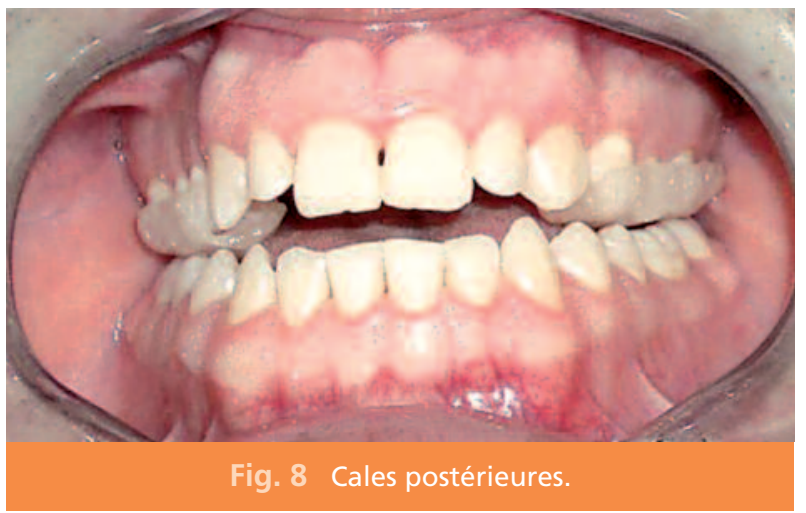

Ces butées sont indiquées en cas de déplacements discaux irréductibles :

- aigus, en permettant le repositionnement de l'ensemble disco-articulaire chez un sujet jeune ;

- chroniques, en favorisant la formation d'un néo-disque et un remaniement osseux autorisant ainsi un jeu fonctionnel et asymptomatique des ATM.

Elles peuvent être également utilisées dans :

- les situations d'urgence avec douleurs intracapsulaires ;

- les dégénérescences du disque avec blocage ;

- les adhérences discales ;

- les claquements articulaires tardifs ;

- les perforations discales ;

- les phénomènes arthrosiques [16].

\section{- Réalisation}

Préalablement à la confection des cales de décompression, la manipulation mandibulaire visant à recoapter le disque articulaire peut être tentée surtout quand celui-ci est déplacé récemment, car les chances de succès sont plus grandes.

La résine est préparée. Quand elle atteint sa phase plastique, une petite quantité est plaquée au niveau des dernières molaires bilatéralement. Le patient, guidé par le praticien, mord dans la position thérapeutique. Une fois la résine durcie, elle est retirée puis ajustée.

La rétention de ce dispositif est obtenue par l'effet d'empreinte dans les reliefs des faces occlusales et des espaces interdentaires.

Les indentations doivent être éliminées pour ne pas bloquer la mandibule dans une position non physiologique.

Comme tous les dispositifs sectoriels, le port doit être de courte durée sans dépasser une quinzaine de jours, pour éviter toute égression dentaire.

\section{> Gouttière de stabilisation}

Quel que soit l'objectif recherché par la gouttière, celle-ci devient au terme des réglages successifs une gouttière de stabilisation de la position thérapeutique obtenue.

Ce type de gouttière est réalisé au laboratoire sur un articulateur semi-adaptable, en position thérapeutique stabilisée et préalablement enregistrée en bouche à l'aide d'un mordu en cire.

Elle présente une surface occlusale légèrement indentée et des contacts occlusaux ponctuels, harmonieusement répartis sur toute l'arcade antagoniste.

Un guide antérieur (incisivo-canin) est établi afin d'organiser les mouvements mandibulaires (propulsion-diduction). 
Elle doit être portée 24 heures sur 24, pendant plusieurs mois, pour régler la position mandibulaire thérapeutique et la maintenir, le temps de procéder au traitement stabilisateur définitif par équilibration soustractive ou additive, prothèse ou orthodontie [17].

\section{> Plan de morsure rétro-incisif}

II se compose d'une plaque palatine retenue par des crochets cavaliers et présentant dans la partie antérieure un épaississement rétro-incisif entraînant une désocclusion des secteurs cuspidés lors de la fermeture buccale.

Ce dispositif libère la mandibule des contraintes verticales et horizontales et des verrous occlusaux constitués par des dents en égression ou un guide antérieur trop abrupt [2].

\section{- Réalisation}

Il est confectionné au laboratoire, en résine acrylique transparente, sur des modèles montés sur articulateur ou bien sur un simple occluseur.

Cet appareillage recouvre les faces palatines des dents jusqu'aux canines pour la plaque de Sved ou jusqu'aux deuxièmes molaires pour la plaque de Jeanmonod.

Il faut que son épaisseur ménage l'espace minimum à une désocclusion postérieure, sans pour autant empiéter sur l'espace libre nécessaire à la phonation, et que sa largeur soit suffisante pour anticiper le recul mandibulaire dû au relâchement musculaire.

Lors de l'ajustage en bouche, le plan rétro-incisif doit être plan, lisse, et établir des contacts suffisants et symétriques avec les dents antagonistes.

Cet appareil doit être porté en permanence y compris la nuit pendant quelques jours (au maximum une semaine).

Après disparition de la symptomatologie douloureuse, le plan de morsure peut être transformé en gouttière de stabilisation par adjonction de résine sur les secteurs cuspidés, recréant un plan lisse et des contacts punctiformes avec les dents antagonistes.

\section{> Gouttière d'urgence : jig de Lucia}

Il a été proposé pour l'enregistrement de la relation centrée par Lucia en 1964.

Ce dispositif interocclusal, ou butée antérieure, supprime l'engrènement cuspidien et entraîne une perte de la mémoire occlusale : déprogrammation neuromusculaire.

Cet appareillage réduit l'activité musculaire des muscles masticateurs de façon temporaire [3]. Il est indiqué en cas de myalgie aiguë ou pour enregistrer la relation centrée. Il est absolument contre-indiqué en cas de troubles articulaires $[5,18]$

\section{- Réalisation}

Il peut être réalisé soit directement en bouche, soit indirectement au laboratoire sur des modèles montés sur articulateur.

Ce dispositif est placé uniquement sur les incisives maxillaires. Le contact avec les incisives mandibulaires se fait selon une surface perpendiculaire en un seul point (fig. 9).

L'épaisseur du jig ménage un espace minimum d'environ $1 \mathrm{~mm}$ au niveau des secteurs cuspidés, en respectant si possible l'espace libre d'inocclusion.

Après son insertion en bouche (fig. 10), on vérifie : la stabilité, la rétention, le contact ponctuel avec l'incisive centrale antagoniste, l'inocclusion postérieure (d'environ $1 \mathrm{~mm}$ ) et la réalisation des mouvements mandibulaires excentrés sans interférences postérieures et sans contraintes au niveau de la butée.

Le jig de Lucia doit être porté uniquement le jour, car il y a un risque de déglutition ou d'inhalation. Il est destiné à un port à court terme (maximum 8 jours) pour éviter toute compression au niveau de l'ATM et toute égression au niveau des secteurs cuspidés. 


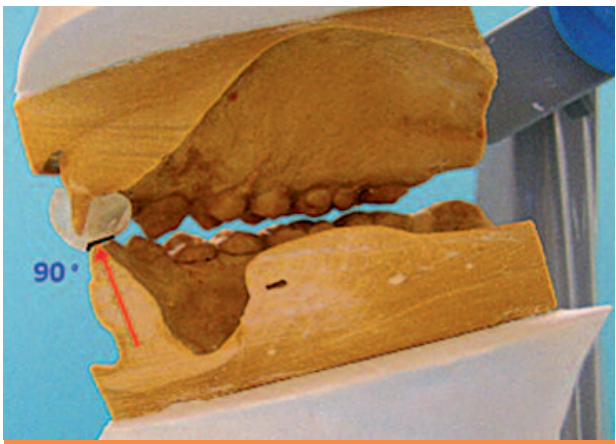

Fig. 9 Orientation de la face palatine de la butée : un seul contact est établi perpendiculairement à I'incisive centrale mandibulaire.

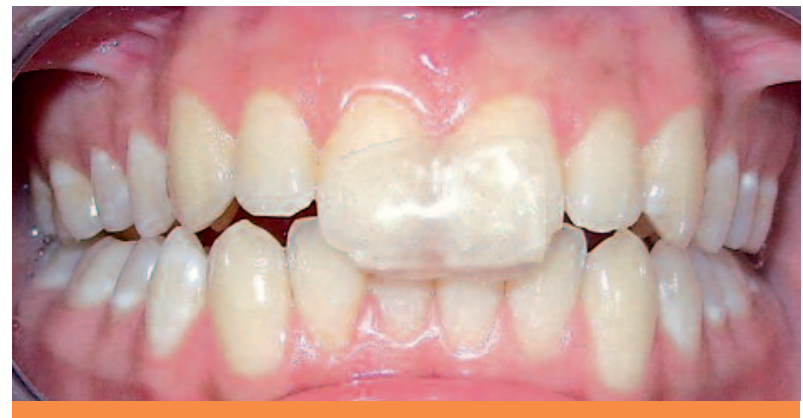

Fig. 10 Jig de Lucia réalisé en résine transparente.

\section{Conclusion}

Les traitements à l'aide de dispositifs interocclusaux se sont révélés être diversement efficaces dans la prise en charge des DTM.

L'utilisation correcte de ces appareillages sousentend l'établissement d'un diagnostic précis prenant en compte les aspects psychosociaux de ces dysfonctionnements [19].

Une fois l'indication de ces dispositifs soigneusement posée, on obtient une sédation objective et durable de la symptomatologie.
En gardant à l'esprit qu'il existe des limites à ces thérapeutiques, il s'agit de bien les connaître pour que le choix soit éclairé.

Le type, la forme et la position de ces différents dispositifs doivent être adaptés de manière spécifique au patient traité. La coopération de ce dernier est indispensable, d'où l'importance des explications données par le praticien pour la compréhension des objectifs recherchés et la bonne observance du traitement.

\section{Bibliographie}

1. Orthlieb J-D, et al. Cadre thérapeutique des dysfonctionnements de l'appareil manducateur. Inf Dent 2004;39:2626-32.

2. Carlier J-F, Ré J-P.

Les dispositifs interocclusaux. Encycl Méd Chir, Odontologie 2008;23-390-A-10.
3. Klasser GF, Greene CS. Oral appliances in the management of temporomandibular disorders.

Oral Surg Oral Med Oral Pathol Oral Radiol Endod 2009;107(2):212-23.

4. Unger F, Giumelli B, Mainetti JL.
Dispositifs interocclusaux. Encycl Méd Chir, Odontologie 1994;23-446-C-10.

5. Orthlieb JD, Gola R, Dufresne JB.

Les gouttières occlusales. Cah Prothèse 1994;87:45-56.

6. Pokorny PH, Wiens J P, Litvak H.

Occlusion for 
fixed prosthodontics:

A historical perspective of the gnathological influence.

J Prosthet Dent

2008;99:299-313.

7. Orthlieb J-D, et al. La relation centrée myostabilisée. Un concept simple, physiologique et consensuel.

Cah Prothèse 2008;141:13-21.

8. Perez $P$.

Apport de l'orthopédie mandibulaire dans le traitement des dérangements des ATM : principes (1re partie). J Edgewise 1996;34:23-38.

9. Glaros AG.

Bruxism.

Behavioral Dentistry 2006;127-37.

10. Dupas PH.

Nouvelle approche du dysfonctionnement cranio-mandibulaire : du diagnostic à la gouttière. Paris : Éditions CdP, 2005.

11. Bodin C, Grazioli L, Abjean J. Altérations fonctionnelles de l'articulation

temporo-mandibulaire.

Encycl Méd Chir, Odontologie 2002:23-435-E-15.

12. Perez $P$.
Apport de l'orthopédie mandibulaire
dans le traitement
des dérangements des ATM :
indications et clinique
(2e partie).
J Edgewise 1996;34:39-51.

13. Badel T, Marotti M, Kern J, Laskarin M.

A quantitative analysis

of splint therapy

of displaced

temporomandibular

joint disc.

Ann Anat

2009;191(9):280-7.

14. Chassagne JF, et al.

Pathologie non traumatique

de l'articulation

temporomandibulaire.

Encycl Méd Chir, Odontologie 2003:23-446-D-10.

15. Rozencweig $\mathrm{D}$.

Plaques

de libération occlusale.

In: Algies et dysfonctionnements

de l'appareil manducateur

(ADAM) :

propositions diagnostiques

et thérapeutiques.

Paris : Éditions CdP,

1994;279-284.

16. Sanchez R, Pouly E.

Détours cliniques.

La décompression de l'ATM.

Les butées postérieures.

Cah Prothèse1998;103:73-8.

17. Lund JP, Lavigne GJ, Dubner R, Sessle BJ.

Douleurs orofaciales.

Des sciences fondamentales

à la pratique clinique.

Quintessence International, 2004.

18. Laplanche $\mathrm{O}$, Pedeutour $\mathrm{P}$, Chossegros $C$.

Traitement de l'urgence des dysfonctionnements de l'appareil manducateur. Rev Odont Stomat 2003;32:135-52.

19. Dworkin SF, Sherman J.

Chronic orofacial pain:

biobehavioral perspectives.

Behavioral Dentistry

2006;99-112.

\section{SUMMARY}

\section{Occlusal appliances and management of TMD}

Anissa ABDELKOUI,

Leila FAJRI,

Ahmed ABDEDINE

\section{Keywords \\ - occlusal appliances \\ - temporomandibular disorders \\ - masticatory system \\ - temporomandibular joint}

Occlusal appliances, originally used to protect teeth and temporomandibular joints in bruxism cases, are currently shown in many clinical situations.

These devices, often used in management of temporomandibular dysfunction (TMD), cause a clear improvement of the condition of patients suffering from these diseases.

Through this article, we try to review the different types of occlusal appliances, to define their roles and goals and then to determine for each the procedure of achievement, in order to make the most suitable choice for the management of TMD. 